\title{
Absorbing Boundary Conditions for Free Surface Waves
}

\author{
J. E. RoMATE \\ Delft Hydraulics, P.O. Box 152,8300 AD Emmeloord, The Netherlands \\ Received November 22, 1989; revised January 29, 1991
}

In this paper the use of absorbing boundary conditions is investigated for the numerical simulation of gravity waves on an incompressible, inviscid fluid in three dimensions. A review of existing methods is given for linear and nonlinear waves, after which first- and second-order partial differential equations are introduced as absorbing boundary conditions for the linearized model. Well-posedness is investigated and it is shown that the reflection properties of the secondorder equation are superior to those of the first-order equation. (c) 1992 Academic Press, Inc.

\section{INTRODUCTION}

In ocean engineering the scattering of waves on fixed and floating bodies is of major importance. Surface waves appear in many forms, from small ripples to large tidal waves, either with small amplitude or being high enough to break. Also, bodies may be small or large compared to the waves; they may have a simple geometry or may be extremely complex. Hence, a broad range of body-wave interactions has to be investigated, necessarily leading to various wave theories and hody-wave models due to the complexity of the Navier-Stokes equations describing the fluid motion.

In general, the compressibility of the water and also the viscous effects are of importance, but for a large class of problems they are negligible and the fluid may be assumed ideal. This is especially the case for large bodies (with dimension typically of the order of the wave length). In this case the Navier-Stokes equations can be simplified considerably by introducing a velocity potential $\phi$ (with velocity $\mathrm{v}=\nabla \phi$ ). The equations describing the flow throughout the fluid domain $\Omega$ then reduce to Laplace's equation for the potential:

$$
\nabla^{2} \phi=0, \quad \mathrm{x} \in \Omega
$$

The equations describing the motion of the free surface $S_{f}$ which bounds this potential flow, are the dynamic and kinematic boundary conditions (see, e.g., Whitham [38]).
These equations are nonlinear and time-dependent:

$$
\begin{aligned}
\frac{D \phi}{D t} & =\frac{1}{2}(\nabla \phi \cdot \nabla \phi)-g z_{f} & & \text { on } S_{f} \\
\frac{D \mathbf{x}_{f}}{D t} & =\mathbf{v}=\nabla \phi & & \text { on } S_{f}
\end{aligned}
$$

where $\mathbf{x}_{f}$ denotes the position of a free surface particle and $z$ is the vertical space coordinate with $z=0$ on the still water level; $g$ is the gravitational acceleration. The above equations are the exact free surface boundary conditions, with the only assumptions that the fluid is ideal and the surface tension is absent.

Mathematically this set of equations can be viewed in different ways. Most naturally it is seen as an elliptic problem with time dependent boundary conditions. Hence, in most numerical procedures it is treated as such: the elliptic Laplace equation is solved at a given time level, after which the time dependent boundary conditions are updated to obtain the proper boundary conditions at the next time level, and so forth. On the other hand, the free surface problem may also be considered as a hyperbolic problem in propagation space, where the "appending" elliptic equation merely causes an integral dependency in space.

Equations (1.1)-(1.3) describe waves of a dispersive nature and form the basis of much of our knowledge on dispersive systems in general. For the linearized equations many analytical results are available, but for the investigation of nonlinear dispersive waves as such or their interaction with surface-piercing bodies, numerical models are indispensable. However, the numerical simulation using the above equations is a difficult task, even in two dimensions, involving complex and time consuming algorithms. Successful schemes in two dimensions were developed by, for instance, Longuet-Higgins and Cokelet [24] and Vinje and Brevig [36]. In both schemes a boundary integral equation technique is used to solve Laplace's equation. Extensions to three dimensions using this technique are presented in, for instance, Isaacson [17] and Romate [30]. In the latter a 
review and extensive literature can be found on the nonlinear problem and the numerical simulation thereof.

One of the problems in the numerical simulation is the reduction of the computational domain. In general, the fluid domain $\Omega$ will extend to infinity in the horizontal directions. In this case a radiation condition at infinity is required to make the problem well-posed. This condition states that the solution corresponds to outgoing waves only, and is known as Sommerfeld's radiation condition [32]. For harmonic solutions of the linearized equations with wave number $k$ it reads:

$$
(k r)^{1 / 2}\left(\phi_{r}-i k \phi\right) \rightarrow 0 \quad \text { as } \quad r \rightarrow \infty
$$

For computational reasons, however, it is necessary to reduce the computational domain to a minimum, and hence the fluid domain $\Omega$ will be truncated at some distance of the area of interest by artificial boundaries. To obtain a wellposed potential problem, boundary conditions are needed on the artificial boundaries as well. Also, since these boundaries are artificial, one would like to have the boundary conditions simulate the behaviour of the excluded part of the fluid domain. For the free surface wave problem this last requirement means that surface wave approaching an artificial boundary should be fully transmitted ("absorbed") at the boundary; i.e., the boundary must be transparent, so that no wave reflections occur.

In this paper the use of partial differential equations as absorbing boundary conditions for the linearized threedimensional model will be considered, as they have been proposed for hyperbolic equations by Engquist and Majda [12]. The investigations will be restricted to the linearized model to facilitate the analysis of the numerical results, but since this analysis is meant to be a basis for future extensions to nonlinear problems, the theoretical development for the linear case will be preceded by a review of existing methods used for both linear and nonlinear free surface wave problems (Section 2).

It will be shown that for the linearized problem the proposed boundary conditions have very favourable reflection properties, given a certain wave frequency and that they will give a well-posed initial boundary value problem (IBVP) in the sense of Kreiss. Numerical results using the proposed model will not be given here, but in the accompanying paper [5].

\section{SURVEY OF ABSORBING BOUNDARY CONDITIONS}

In the literature several methods have been proposed to absorb free surface waves, and some of these are reviewed in, e.g., $[40,29]$. A short description of each of these methods will be given.

\section{Periodic Boundary Condition}

This technique has been favoured in most two-dimensional nonlinear models. The solution is assumed to be periodic in space, so that the values of the unknowns on one vertical boundary can be set equal to those on the other vertical boundary of the domain. Advantages: very simple to implement, and artificial boundaries can be chosen at very short distances. Disadvantages: limited application due to requirement of periodicity. Longuet-Higgins and Cokelet [24] used periodicity for their boundary integral method to be able to transform the free surface to a circle.

\section{Artificial Damping (Sponge Layers)}

Another possibility of simulating an infinite outer field at finite distance, is the use of artificial damping, in particular in the form of a sponge layer. In this method an artificial dissipative term is added -implicitly or explicitly - to the equations near the artificial boundaries of the truncated domain, so that outgoing waves are asborbed with as little wave reflection as possible.

For free surface flow this technique was used by, e.g., Chan [6], who added a linear damping term to the momentum equations, in his $2 \mathrm{D}$ finite difference scheme. Other examples of the use of sponge layers can be found in, for instance, Larsen and Dancy [20], and Israeli and Orszag [18]. In both articles the authors use sponge layers in combination with partial differential equations to absorb outgoing waves.

In combination with an integral equation method to solve the potential flow, the damping term must be added to the free surface boundary conditions. Baker, Meiron, and Orszag [1] added a dissipative term to both the kinematic and the dynamic boundary condition (in their case equations for the surface wave elevation $\eta$ and the vortex sheet strength $\gamma$ ). Specifically,

$$
\frac{\partial \eta}{\partial t}=\mathrm{RHS}-v(x)(\eta-h)
$$

and

$$
\frac{\partial \gamma}{\partial t}=\mathrm{RHS}-v(x) \gamma,
$$

where $v(x)$ is the damping coefficient and RHS is the righthand side of their original equations. The damping coefficient was chosen to be quadratic in $x$ so that the extra term and its derivative vanish at the point where the damping zone meets the computational domain.

Betts and Mohamad [3] added the dissipative term only to the dynamic boundary condition in their 2D nonlinear model:

$$
\frac{D \phi}{D t}-\frac{1}{2}(\nabla \phi)^{2}+g z+v \phi=0 .
$$




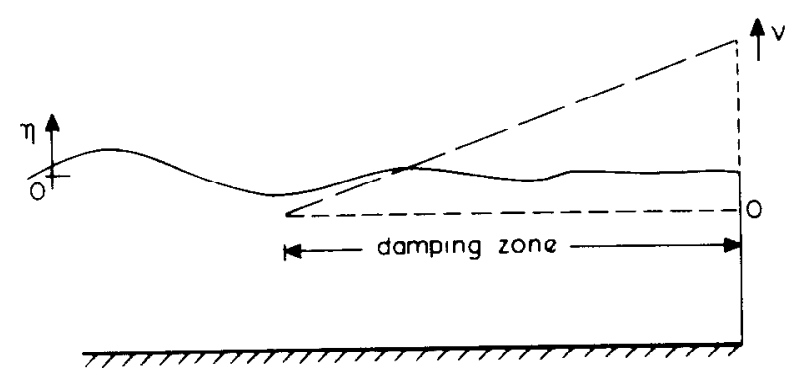

FIG. 1. Damping zone with linearly increasing $v$.

The Rayleigh damping term $v \phi$ was specified nonzero in the damping zone; it increases linearly from zero at the beginning of the damping zone, to a given positive value at the end of the zone (Fig. 1).

They studied the reflection properties for different values of $v$. For small values of $v$ there is little dissipation and waves reflect almost entirely at the artificial boundary at the end of the damping zone. For large values of $v$ the absorbing area itself will act as a boundary, and waves reflect at the beginning of the damping zone. An optimal value can be found somewhere in between. Betts and Mohamad found an optimal value of $v=0.4$ resulting in $6 \%$ reflection when using a damping zone that was one wavelength long.

Advantages of this method: easy to implement, good reflection properties for a wide range of frequencies. Disadvantages: a fairly large domain is needed for the damping zone (in the order of one wave length).

\section{Simple Far Field Solutions}

Another possibility of absorbing waves at an artificial boundary is the use of simple outer field solutions. In the outer field boundaries and boundary conditions are simplified such that analytical solutions can be found in closed form, usually expressed as eigenfunction expansions. At the artificial boundary the analytical solution is then matched to the solution of the computational domain. An example of this technique is the so-called hybrid integral equation method. It is described in, e.g., Liu and Abbaspour [23] for the $2 \mathrm{D}$ case, where they study the diffraction of waves by an infinite cylinder. They solve the potential problem in the interior with a boundary integral equation method and use an analytical solution for the outer domain satisfying the linearized free surface conditions, and Sommerfeld's radiation condition at infinity $[32,2]$ :

$$
\begin{aligned}
\phi_{a}(x, t)= & A_{0} \cdot \frac{\omega}{k} \cdot \frac{\cosh (k(z+h))}{\sinh (k h)} \cdot e^{i(k x-\omega t)} \\
& +\sum_{n=1}^{\infty} A_{n} \cdot \frac{\cos \left(\kappa_{n}(z+h)\right)}{\cos \left(\kappa_{n} h\right)} \\
& \cdot e^{\kappa_{n} x-i \omega t} .
\end{aligned}
$$

The unknown coefficients $A_{0}$ and $A_{n}$ in the expansion are the amplitudes of the propagating and evanescent modes in the solution (see next section) and are determined by the matching at the artificial boundary.

Finnigan and Yamamoto [13] use this technique for a porous breakwater problem (linear, 2D), Nestegard and Sclavounos [26] for a deep water time-harmonic radiation problem (also 2D), and Lin, Newman, and Yue [22] use a linear outer solution for nonlinear 2D computations in the interior. Dommermuth and Yue [10] use an axisymmetric solution in the exterior, and Tsay and Liu [33] combine the linear outer solution with a finite element method in the interior.

These are but a few examples of the many papers that have been published. Advantages of the method: for linear inner solutions good accuracy and, also, evanescent modes are absorbed. Disadvantages are: more difficult to implement, and extension to time domain problems with more frequencies is complicated.

\section{Differential Equations Matching the Outer Solution}

In the previous technique explicit expressions of the outer domain solutions were used for matching at the artificial boundary. Bayliss, Gunzburger, and Turkel [2] use the eigenfunction expansions of the outer domain to derive a set of linear partial differential equations which can be imposed at finite distance on an artificial boundary. The operators annihilate the first $m$ terms of the expansion and thus replace in an implicit manner these explicit expressions. The lowest order operator of this set of radiation conditions is Sommerfeld's equation, but here it is applied at the boundary.

\section{Sommerfeld-Orlanski}

An interesting approach for imposing Sommerfeld's condition on artificial boundaries was published by Orlanski [27]. The novelty in his approach was that the phase velocity needed in this condition was evaluated numerically in the vicinity of the boundary. The main advantage of this method is therefore that one does not need to know in advance the frequency of an approaching wave. Orlanski used this method in a finite difference scheme for hyperbolic flow, and it was Chan [7] who was the first to apply it to a free surface flow problem.

Yen and Hall [39] examined several schemes to improve the implementation of Chan. They also used Sommerfeld's condition for both the wave elevation $\eta$ and the potential $\phi$ in their finite difference scheme.

Finally, in their boundary element method Lennon, Liu, and Liggett [21] avoided the calculation of the unknown $c$ by using $c=\sqrt{g h}$, i.e., the phase velocity of shallow water waves. Romate [29] studied the reflection properties of Sommerfeld's condition for various choices of $c$, and 
Vichnevetsky and Pariser [35] considered higher order discretizations of Sommerfeld's condition.

Sommerfeld-Orlanski is probably the most widely used technique. The condition is simple to implement and, in general, the results are fairly good.

\section{Partial Differential Equations}

Sommerfeld's radiation condition and the absorbing conditions of Bayliss et al. are two examples of this category, but these have been listed separately because of their widespread use. Sommerfeld's condition is a first-order equation, and the conditions of Bayliss et al. are higher order generalizations to absorb spherical waves.

However, higher order generalizations of Sommerfeld's equation, to absorb plane waves in the potential free surface model used here, are seldom used. Most of the theory has been developed for hyperbolic waves $[11,12]$, and has to be modified to be applicable to free surface models. For the (hyperbolic) nonlinear shallow water equations it was used by, e.g., Verboom [34]. Israeli and Orszag [18] compared this technique with the use of sponge layers.

The main advantage of this technique is that the artificial boundaries can be taken very close to the area of interest. Also it is fairly easy to predict reflection properties. In the next sections the use of these partial differential equations will be investigated.

\section{LINEARIZED FREE SURFACE WAVES}

In case of small amplitude waves, the nonlinear free surface conditions may be linearized in the usual way: nonlinear terms in $\phi$ and $\eta$ are neglected, and the conditions are imposed on the still water level. Introducing an artificial boundary of infinite length at $x=0$, we want to consider the solution of the resulting linear model for $x \leqslant 0$, given by the following equations:

$\nabla^{2} \phi=0 \quad$ in $\quad \Omega=\{\mathbf{x} \mid x<0, y \in \mathbb{R},-h<z<0\}$,

$\frac{\partial \eta}{\partial t}=-\frac{\partial \phi}{\partial n} \quad$ on $\quad z=0$

$\frac{\partial \phi}{\partial t}=-g \cdot \eta \quad$ on $\quad z=0$,

$\frac{\partial \phi}{\partial n}=0 \quad$ on $\quad z=-h$.

The bottom will be taken horizontal.

The typical wave phenomena of linear waves occur in $(x, y)$-space, which will be referred to as the propagation space. The dispersion relation of this free surface problem,

$$
\omega^{2}=g \cdot k \cdot \tanh (k h)
$$

relates the frequency $\omega$ and the wave number $k$ and has two real roots $\pm k$, leading to propagating waves (with wave velocity $c=\omega / k$ ) and, also, an infinite number of imaginary solutions $k= \pm i \kappa_{n}$. The corresponding solutions are the evanescent modes [25]. The evanescent modes are not absorbed by the boundary conditions introduced hereafter [28], but boundary conditions for these modes can also be derived.

To derive absorbing boundary conditions for propagating waves, we need the equations describing the wave propagation in $(x, y)$-space. These can be obtained by eliminating the $z$-dependency in Eq. (3.1)-(3.4), resulting in

$$
\phi(\mathbf{x}, t)=B \cdot \cosh (k(z+h)) \cdot \tilde{\phi}(\tilde{\mathbf{x}}, t)
$$

with $\tilde{\mathbf{x}}=(x, y)^{\mathrm{T}}$, and the equations

$$
\begin{aligned}
& \nabla^{2} \tilde{\phi}+k^{2} \tilde{\phi}=0, \\
& \frac{\partial^{2} \tilde{\phi}}{\partial t^{2}}+g \cdot k \cdot \tanh (k h) \cdot \tilde{\phi}=0, \\
& \quad \text { in } \quad \tilde{\Omega}=\{\tilde{\mathbf{x}} \mid x<0, y \in \mathbb{R}\}
\end{aligned}
$$

for the dependent variable $\bar{\phi}(x, y)$ in propagation space. This set of equations has solutions of the form

$$
\tilde{\phi}(\tilde{\mathbf{x}}, t)=e^{i(\mathbf{k} \cdot \tilde{\mathbf{x}}-\omega t)} .
$$

For the investigations on the absorbing boundary conditions, an incident wave will be used, describing by the velocity potential

$$
\phi_{i}(\mathbf{x}, t)=B \cdot \cosh (k(z+h)) \cdot \widetilde{\phi}_{i}(\tilde{\mathbf{x}}, t),
$$

with (see Fig. 2)

$$
\begin{aligned}
\tilde{\phi}_{r}(\tilde{\mathbf{x}}, t) & =e^{i\left(-\omega t+\mathbf{k}_{i} \cdot \tilde{\mathbf{x}}\right)} \\
& =e^{i(-\omega t+k[\cos (\theta) x+\sin (\theta) v])}
\end{aligned}
$$

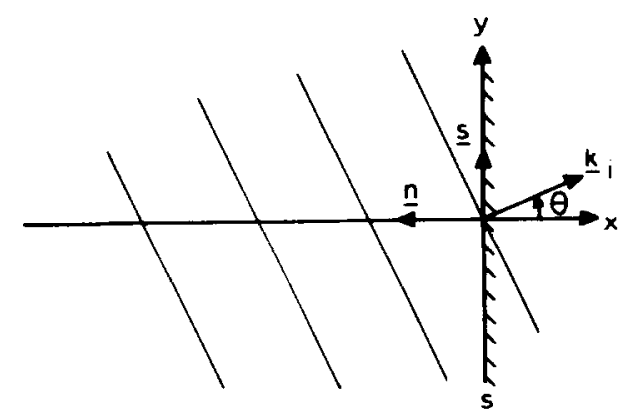

FIG. 2. Plane incident wave propagation in $\mathbf{k}_{i}$-direction. 
As the incident wave strikes the absorbing boundary $S$, reflection will occur. The reflected wave is given by

$$
\begin{aligned}
\tilde{\phi}_{r}(\tilde{\mathbf{x}}, t) & =\operatorname{Re}^{i\left(-\omega t+\mathbf{k}_{r} \cdot \tilde{\mathbf{x}}\right)} \\
& =\operatorname{Re}^{i(-\omega t+k[-\cos (\theta) x+\sin (\theta) y]} .
\end{aligned}
$$

If a perfectly absorbing boundary condition is used on $S$, no energy will be reflected into $\tilde{\Omega}=\{(x, y) \mid x<0, y \in \mathbb{R}\}$, i.e., $R=0$.

In numerical computations only approximate absorbing boundary conditions can be used. In two papers Engquist and Majda [11,12] proposed a theory for developing a hierarchy of absorbing boundary conditions for a hyperbolic equation to be used on artificial boundaries. With the use of the dispersion relation they factorize the equation into first-order equations describing the propagation of waves along bicharacteristics. For the wave equation, for instance, this means that a resulting first-order equation describes either a left going or a right going wave but does not admit the other. Using the appropriate of these firstorder equations as a boundary condition, they arrive at an exact boundary condition absorbing waves of all frequencies, from all directions. The problem, however, is that these first-order equations are not partial differential equations but pseudodifferential equations, which are not local in time or space. Therefore Engquist and Majda introduce Padéapproximants of the matching dispersion relation to obtain local partial differential equations as absorbing boundary conditions. This idea has been developed further by many authors, e.g., by Wagatha [37], and Higdon [15]. The latter presents an extension to absorb waves from different directions, and this idea will be applied here.

\section{FIRST-ORDER EQUATIONS}

Consider the following first-order differential equations on $x=0$ :

$$
\begin{gathered}
L \tilde{\phi}=\left.\left(\frac{\partial}{\partial t}+c \frac{\partial}{\partial r}\right) \tilde{\phi}\right|_{x=0}=0, \\
c=\frac{\omega}{k}, \quad \mathbf{r}=\left(\begin{array}{c}
\cos \alpha \\
\sin \alpha
\end{array}\right) .
\end{gathered}
$$

This condition absorbs the given incident wave if $\alpha=\theta$ (only $|\alpha| \leqslant \pi / 2$ will be considered). With

$$
\frac{\partial}{\partial r}=\mathbf{r} \cdot \nabla=\cos (\alpha) \frac{\partial}{\partial x}+\sin (\alpha) \frac{\partial}{\partial y},
$$

(4.1) can be written as

$$
\mathrm{BC} 1: \frac{\partial \tilde{\phi}}{\partial t}+\left.c\left(\cos (\alpha) \frac{\partial \tilde{\phi}}{\partial x}+\sin (\alpha) \frac{\partial \tilde{\phi}}{\partial y}\right)\right|_{x=0}=0
$$

The reflection coefficient $R$ is found by substituting $\tilde{\phi}_{i}+\tilde{\phi}_{r}$ into (4.3):

$$
\begin{aligned}
R_{\mathrm{BC} 1} & =\frac{-1+\cos (\alpha) \cos (\theta)+\sin (\alpha) \sin (\theta)}{1+\cos (\alpha) \cos (\theta)-\sin (\alpha) \sin (\theta)} \\
& =-\frac{1-\cos (\alpha-\theta)}{1+\cos (\alpha+\theta)}
\end{aligned}
$$

A second first-order boundary condition that absorbs incident waves at angle $\alpha$ with the normal is

$$
\mathrm{BC} 2: \cos (\alpha) \frac{\partial \tilde{\phi}}{\partial t}+\left.c \frac{\partial \tilde{\phi}}{\partial x}\right|_{x=0}=0 .
$$

The reflection coefficient is now given by

$$
R_{\mathrm{BC} 2}=-\frac{\cos (\alpha)-\cos (\theta)}{\cos (\alpha)+\cos (\theta)}
$$

If we compare the magnitude of the reflection coefficients of the two alternatives (4.3) and (4.5), neither of the conditions has an important advantage over the other. They are approximately the same in magnitude for any combination of $\alpha$ and $\theta$.

If $\alpha=0$ (perfect absorption for waves at normal incidence) the conditions are exactly the same, and thus have the same reflection coefficient for any $\theta$. On the other hand, if $\alpha=\pi / 2$ (tangential incidence) the equations are very different, and their respective reflection coefficients are, although of the same magnitude, of opposite sign. Equation (4.3) becomes

$$
\frac{\partial \tilde{\phi}}{\partial t}+\left.c \frac{\partial \tilde{\phi}}{\partial y}\right|_{x=0}=0
$$

with $R=-1$ for $\theta \neq \pi / 2$, and Eq. (4.5) reduces to

$$
\left.\frac{\partial \widetilde{\phi}}{\partial x}\right|_{x=0}-0
$$

with $R=1$ for $\theta \neq \pi / 2$.

In practically all publications equation (4.5), not (4.3), is used. (See, for instance, $[11,12,34,39]$ for examples on the use of (4.5.) In Section 3.6 we will see that indeed (4.5) is to be preferred.

\section{SECOND-ORDER EQUATIONS}

The first-order equations given above are very well suited when incident waves are involved from only one known direction. However, it is obvious that in most applications 
either the direction is unknown in advance, so that an optimum value of $\alpha$ can only be guessed, or that waves approach the boundary from more than one direction and any choice of $\alpha$ will lead to reflection.

Therefore, second-order differential equations will now be considered, to improve on the reflection properties of the first-order equations given above.

Equation (4.1) annihilates waves (with phase velocity $c$ ) approaching the boundary at an angle $\alpha$. To annihilate waves from $n$ directions an $n$th order differential equation is introduced by applying the operator $L n$ times, with different choices of $\alpha(|\alpha| \leqslant \pi / 2)$ :

$$
\begin{aligned}
& \left.\prod_{i=1}^{n}\left(\frac{\partial}{\partial t}+c \frac{\partial}{\partial r_{i}}\right) \tilde{\phi}\right|_{x=0}=0, \\
& c=\frac{\omega}{k}, \quad \mathbf{r}_{i}=\left(\begin{array}{c}
\cos \alpha_{i} \\
\sin \alpha_{i}
\end{array}\right) .
\end{aligned}
$$

In most applications only two main directions are involved, and therefore we will continue with $n=2$. Now, using (4.5) as an approximation of (4.1), and choosing two directions $\alpha_{i}(i=1,2)$, we have the following second-order absorbing boundary condition:

$$
\mathrm{BC} 3:\left.\prod_{i=1}^{2}\left(\cos \left(\alpha_{i}\right) \frac{\partial}{\partial t}+c \frac{\partial}{\partial x}\right) \tilde{\phi}\right|_{x=0}=0
$$

Here it is assumed that the waves from the two directions have the same frequency $\omega$, and therefore the same phase velocity $c$. The reflection coefficient $R$ is now given by

$$
R_{\mathrm{BC} 3}=-\prod_{i=1}^{2}\left(\frac{\cos \left(\alpha_{i}\right)-\cos (\theta)}{\cos \left(\alpha_{i}\right)+\cos (\theta)}\right) .
$$

Equation (5.2) involves the second normal derivative $\partial^{2} \bar{\phi} / \partial x^{2}$. However, since (5.2) will be used as a boundary condition of Laplace's equation, this second derivative should be expressed in terms of the function $\tilde{\phi}$ itself, the first normal derivative, the time derivatives and/or the tangential derivatives. This could be done by using (3.7), but in Section 6 it will become clear that this choice might give problems in actual computations. Hence a combination of Eq. (3.7) and (3.8) will be used.

Subtracting Eq. (3.8) from $D$ times (3.7), gives the equation:

$$
-\frac{\partial^{2} \tilde{\phi}}{\partial t^{2}}+D \cdot \nabla^{2} \tilde{\phi}-\left(\omega^{2}-k^{2} D\right) \cdot \tilde{\phi}=0
$$

This equation is a Klein-Gordon equation, if $\omega^{2}-k^{2} D>0$, and has solutions of form (3.9). The Klein-Gordon equation is a hyperbolic equation, but also falls into the dispersive class (see Whitham [38]).

Choosing $D=c^{2}$ in (5.4) reduces this equation to the wave equation. But, also, the wave equation cannot be used as the field equation for the waves in propagation space, because it admits solutions which are lower order polynomials in $x, y$, and $t$. Some of these solutions may grow in time and spoil the solution. Therefore a different value of $D$ will be used: $D=c c_{g}$ (where $c_{g}$ is the group velocity, defined by $\left.c_{g}=\partial \omega / \partial k\right)$. This results in a special case of the mildslope equation, namely for a horizontal bottom (see [9]).

Hence, with

$$
\frac{\partial^{2} \tilde{\phi}}{\partial x^{2}}=\frac{1}{c c_{g}} \frac{\partial^{2} \tilde{\phi}}{\partial t^{2}}-\frac{\partial^{2} \tilde{\phi}}{\partial y^{2}}+\left(\frac{\omega^{2}}{c c_{g}}-k^{2}\right) \tilde{\phi},
$$

(5.2) can be written as

$$
\mathrm{BC} 4: a \cdot \frac{\partial^{2} \tilde{\phi}}{\partial t^{2}}+b \cdot \frac{\partial}{\partial t} \frac{\partial \Phi}{\partial x}-c^{2} \cdot \frac{\partial^{2} \tilde{\phi}}{\partial y^{2}}+\left.e \cdot \tilde{\phi}\right|_{x=0}=0
$$

with

$$
\begin{aligned}
& a=\cos \left(\alpha_{1}\right) \cos \left(\alpha_{2}\right)+c / c_{g}, \\
& b=c \cdot\left(\cos \left(\alpha_{1}\right)+\cos \left(\alpha_{2}\right)\right), \\
& e=c^{2}\left(\frac{\omega^{2}}{c c_{g}}-k^{2}\right) .
\end{aligned}
$$

\section{WELL-POSEDNESS OF THE HALF-SPACE PROBLEM}

Laplace's equation requires one boundary condition per boundary of the fluid domain and hence also one condition on each artificial boundary. However, this condition should render the problem well-posed, also as a time-dependent problem. Therefore we will investigate the well-posedness of the initial boundary value problem (IBVP) formulated in the previous sections with the proposed absorbing boundary conditions as boundary conditions of a half-space problem.

First some of the main results for hyperbolic systems will be given, as described in the review of Higdon [16]. We will follow his notation most of the time. See also [14]. A definition of well-posedness is given by Hadamard:

Definition 1. Well-posedness (Hadamard). An IBVP is well-posed if for all $\mathbf{x} \in \Omega, t \geqslant 0$, a solution exists, which furthermore is unique and depends continuously on the initial and boundary data given.

In practical cases this definition is of little use. However, for linear hyperbolic equations we can reformulate this into a more practical definition. 
Definition 2. Well-posedness (Kreiss). Consider the hyperbolic system

$$
\mathbf{u}_{t}=A \mathbf{u}_{x}+\sum_{j=1}^{m} B_{j} \mathbf{u}_{y_{j}}+C \mathbf{u}+\mathbf{d}
$$

for $t>0$, and $\mathbf{x} \in \Omega=\left\{\mathbf{x}=(x, \mathbf{y}) \mid x<0, y_{j} \in \mathbb{B}\right\}$. $\mathbf{u}$ is a vector with $n$ components. With initial condition

$$
\mathbf{u}(x, \mathbf{y}, 0)=f(x, \mathbf{y}), \quad(x, \mathbf{y}) \in \Omega
$$

and boundary condition

$$
E \mathbf{u}(0, \mathbf{y}, t)=g(\mathbf{y}, t), \quad(\mathbf{y}, t) \in \partial \Omega \times[0, T],
$$

this problem is well-posed if for smooth coefficients and data the solution shows bounded growth, i.e., that its norm can be estimated in terms of $\mathbf{d}, f$, and $g$ (energy estimate).

The energy estimate in terms of Sobolev norms is given in [16]:

$$
\begin{aligned}
\|\mathbf{u}\|_{\Omega \times[0, T]}+\|\mathbf{u}\|_{\partial \Omega \times[0, T]}+\|\mathbf{u}\|_{\Omega} \\
\quad \leqslant K\left(\|f\|_{\Omega}+\|g\|_{\partial \Omega \times[0, T]}+\|\mathbf{d}\|_{\Omega \times[0, T]}\right) .
\end{aligned}
$$

$K$ is independent of $\mathbf{u}, \mathbf{d}, f$, and $g$.

For more general smooth boundaries, the problem can be written in terms of the half-space problem defined above, by mapping the boundary locally onto the tangent plane at each point of the boundary.

In order to determine a unique solution of the hyperbolic system, boundary conditions are needed. However, the choice and number of boundary conditions is not trivial, and an arbitrary choice can easily render the problem illposed. Sometimes choices are clear from physical interpretations, but usually this is not the case.

To determine how many boundary conditions are needed on a given boundary one can use the method of characteristics. In more space dimensions this method gives necessary conditions for the problem to be well-posed. It states that:

THEOREM 1 (Necessary condition well-posedness). For well-posedness of the system (6.1)-(6.2) it is necessary that the number of boundary conditions at a point on the boundary is equal to the number of ingoing characteristics at that point (see Gerritsen [14]).

In one space dimension (but not in more space dimensions) this condition is also sufficient for the problem to be well-posed. In more dimensions two methods can be used to determine the well-posedness of a problem: the "energy method" (see $[8,14]$ ), and the "normal mode analysis," developed by Kreiss [19] and others. Both methods give necessary and sufficient conditions under certain restrictions.

We will use the "normal mode analysis," which applies to strictly hyperbolic and symmetric hyperbolic systems and equations. Strictly hyperbolic means that all eigenvalues of the characteristic equation are imaginary and distinct, and symmetric hyperbolic means that the matrices $A$ and $B_{j}$ in (6.1) are hermitian. It also assumes that the plane $x=0$ is noncharacteristic.

The problem is Fourier-transformed in $\mathbf{y}$ and Laplacetransformed in $t$; i.e., solutions of the form

$$
e^{\xi x+i \eta \cdot y+\tau t}
$$

are considered, where $\xi$ is obtained from the dispersion relation of (6.1).

Now, a sufficient condition for well-posedness of (6.1)-(6.3) is given by the uniform Kreiss condition, which states that solutions of the form (6.5) which are purely harmonic in $\mathbf{y}$-direction and bounded in the entire domain, may not grow in time. More formally:

THEOREM 2. (Sufficicnt condition well-posedness, uniform Kreiss condition). The half-space problem (6.1)-(6.3) is well-posed if, after Fourier-transforming the problem in the $\mathbf{y}$-direction with dual variable $\mathbf{h}$, and Laplace-transforming it in the $t$-direction with dual variable $\tau$, the following criterion is satisfied:

$$
|B(\eta, \tau)| \geqslant \varepsilon>0, \quad \operatorname{Re}(\tau) \geqslant 0, \quad \operatorname{Im}\left(\eta_{i}\right)=0,
$$

where $B(\eta, \tau)$ is the transformed boundary condition (6.3), and only solutions of the form (6.5) are considered with $\operatorname{Re}(\xi) \geqslant 0$.

It is noted that the valuc of $\xi$ for $\operatorname{Rc}(\tau)=0$ is defined to be the limit of values of $\xi$ corresponding to $\operatorname{Re}(\tau)>0$. If the UKC is violated only for a value of $\tau$ with $\operatorname{Re}(\tau)=0$, then the problem is said to be weakly ill-posed: disturbances will neither grow nor decay in time. This value of $\tau$ is called a "generalized eigenvalue." Mild instabilities can be present in the solution, but according to Higdon [15] this disadvantage is outweighed by the advantage of having small reflection coefficients for the absorbing boundary conditions of order two or less.

We will now apply this theorem to the given equations, to determine which boundary conditions render the IBVP well-posed. In Eq. (5.4) the field equations (3.7) and (3.8) were rewritten as a Klein-Gordon equation for use in the boundary conditions. Therefore this equation will be taken as the field equation (with $D=c c_{g}$ ):

$$
\begin{gathered}
-\frac{\partial^{2} \tilde{\phi}}{\partial t^{2}}+c c_{g} \nabla^{2} \tilde{\phi}-\left(\omega^{2}-k^{2} c c_{g}\right) \tilde{\phi}=0, \\
\tilde{\mathbf{x}} \in \tilde{\Omega}, t>0 .
\end{gathered}
$$

It can be written as a first-order system by the substitution

$$
u_{1}=\bar{\phi}, \quad u_{2}=\bar{\phi}_{t}, \quad u_{3}=\bar{\phi}_{x}, \quad u_{4}=\bar{\phi}_{y},
$$


resulting in a symmetrizable hyperbolic, but not strictly hyperbolic system. The characteristic equation has two zero eigenvalues, due to the fact that (6.8) leads to a fourth-order system which is not fully equivalent to (6.7) (see also Section 5.2 of [38]), and two nonzero eigenvalues $\sqrt{c c_{g}}$ and $-\sqrt{c c_{g}}$, showing that one boundary condition is needed in each $\mathrm{x}$ on $S$.

Now, for the normal mode analysis, consider solutions

$$
e^{\xi x+i \eta y+\tau t}
$$

of the Klein-Gordon equation. In (6.9) $\xi, \eta$, and $\tau$ are complex numbers, and it is assumed that $\operatorname{Re}(\xi) \geqslant 0, \operatorname{Im}(\eta)=0$, and $\operatorname{Re}(\tau) \geqslant 0$. The dispersion relation is

$$
\tau^{2}=c c_{g}\left(\xi^{2}-\eta^{2}\right)-\left(\omega^{2}-k^{2} c c_{g}\right)
$$

or

$$
\xi=\left[\eta^{2}+\tau^{2} / c c_{g}+\left(\omega^{2} / c c_{g}-k^{2}\right)\right]^{1 / 2},
$$

where the root with non-negative real part is chosen. Note that $\eta^{2}, c c_{g}$, and $\left(\omega^{2} / c c_{g}-k^{2}\right)$ are all real and non-negative.

Now, let

$$
\left.L\left(\frac{\partial}{\partial x}, \frac{\partial}{\partial y}, \frac{\partial}{\partial t}\right) \tilde{\phi}\right|_{x=0}=0
$$

be the homogeneous boundary condition; then the UKC demands that $L(\xi(\eta, \tau), i \eta, \tau) \neq 0$ for well-posedness.

BC1. For boundary condition BC1, Eq. (4.3), we have

$$
\begin{aligned}
L(\eta, \tau)= & \tau+c \cdot \cos (\alpha)\left[\eta^{2}+\tau^{2} / c c_{g}\right. \\
& \left.+\left(\omega^{2} / c c_{g}-k^{2}\right)\right]^{1 / 2}+c \cdot \sin (\alpha) i \eta .
\end{aligned}
$$

If $\alpha= \pm \pi / 2$, then $L=0$ for $\tau= \pm c \eta i$, i.e., a generalized eigenvalue exists, and the problem is weakly ill-posed.

Now consider the case $|\alpha|<\pi / 2$. If $\operatorname{Re}(\tau)>0$, then $\operatorname{Re}(L(\eta, \tau))>0$ and hence the UKC is satisfied. If $\tau=0$ we have $L \neq 0$. For $\tau=\beta i$ and $\tau \neq 0$, discr $=\eta^{2}-\beta^{2} / c c_{g}+$ $\left(\omega^{2} / c c_{g}-k^{2}\right)$ is real, and we have

discr $>0: \xi$ is real and positive, and $\operatorname{Re}(L)>0$.

discr $=0: \xi$ is zero; $L=0$ for $\sin (\alpha)=-\beta / c \eta$. Again we have a generalized eigenvalue.

$\operatorname{discr}<0: \xi= \pm i \gamma \quad(\gamma>0)$. Considering $\lim \operatorname{Re}(\tau) \downarrow 0$, with $\operatorname{Re}(\xi) \downarrow 0$ also, we see that we only have to consider $\xi=+i \gamma$ as the limiting case (incoming mode) for $\beta>0$, and $\xi=-i \gamma$ for $\beta<0$. However, also in this case generalized eigenvalues exist.

Thus in general, BC1 renders the problem weakly ill-posed.
BC2. For boundary condition BC2, Eq. (4.5), we have

$$
\begin{aligned}
L(\eta, \tau)= & \cos (\alpha) \tau+c \\
& \cdot\left[\eta^{2}+\tau^{2} / c c_{g}+\left(\omega^{2} / c c_{g}-k^{2}\right)\right]^{1 / 2}
\end{aligned}
$$

It is easily shown that $\mathrm{BC} 2$ renders the problem well-posed for $|\alpha|<\pi / 2$, and weakly ill-posed only for $|\alpha|=\pi / 2$ (the case of a solid wall boundary condition).

BC.3. For boundary condition BC3, Eq. (5.2), we have

$$
\begin{aligned}
L(\eta, \tau)= & \prod_{i=1}^{2}\left(\cos \left(\alpha_{i}\right) \tau+c\right. \\
& \left.\cdot\left[\eta^{2}+\tau^{2} / c c_{g}+\left(\omega^{2} / c c_{g}-k^{2}\right)\right]^{1 / 2}\right) .
\end{aligned}
$$

$L=0$ if one of the factors is zero. Therefore, the results of BC2 apply here for $\alpha=\alpha_{i}$. Thus it may be concluded that BC3 renders the problem well-posed if $\left|\alpha_{i}\right|<\pi / 2(i=1,2)$. If $\alpha_{1}=\pi / 2$ or $\alpha_{2}=\pi / 2$ (or both), then the problem is weakly ill-posed.

BC4. For boundary condition BC4, Eq. (5.6), we have the same results as for $\mathrm{BC} 3$.

Thus, all boundary conditions render the problem weakly ill-posed for $\alpha= \pm \pi / 2$. This choice of $\alpha$ represents a solid wall or, in general, a boundary that fully reflects waves of normal incidence.

Of the two first-order boundary conditions $\mathrm{BC} 1$ and $\mathrm{BC} 2$ the latter is to be preferred for two reasons: it renders the problem well-posed for $|\alpha|<\pi / 2$, whereas $\mathrm{BC} 1$ does not, and for $|\alpha|=\pi / 2$ the problem is weakly ill-posed only for waves of tangential incidence $(\xi=0)$ so that disturbances are confined to the boundary and will not propagate into the interior. Using $\mathrm{BC} 1$, the problem is also weakly ill-posed for waves of non-tangential incidence. For $\mathrm{BC} 3$ and $\mathrm{BC} 4$ the results of BC2 apply.

Finally, it is noted that the use of the wave equation, or of Eq. (3.7) to eliminate the second normal derivative in (5.2) would lead to second-order boundary conditions which render the IBVP weakly ill-posed also for $\left|\alpha_{i}\right|<\pi / 2$ (cf. BC4), so that errors are free to propagate from the boundary into the interior. Hence BC4 is preferred.

\section{THE INFLUENCE OF CORNERS}

In the previous sections we examined the well-posedness of the half-space problem (6.7) with one of the boundary conditions $\mathrm{BC} 1-\mathrm{BC} 4$ imposed on the infinite boundary $x=0$.

In practical calculations, however, the computational domain is finite. Therefore the boundaries of the computational domain are also of finite length. To investigate the 


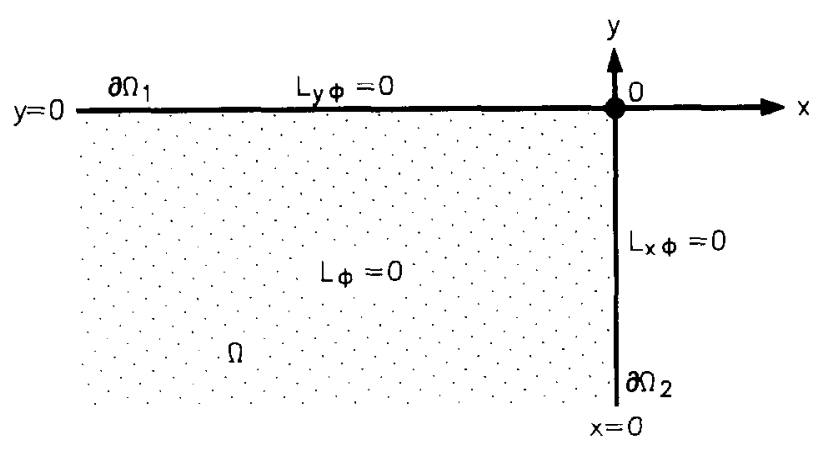

FIG. 3. A hyperbolic quarter-space problem.

influence of finite boundaries, that is, of corners, consider the quarter-space problem (Fig. 3),

$$
\begin{aligned}
& -\frac{\partial^{2} \tilde{\phi}}{\partial t^{2}}+c c_{g} \nabla^{2} \tilde{\phi}-\left(\omega^{2}-k^{2} c c_{g}\right) \tilde{\phi}=0, \\
& \quad x<0, y<0, t>0, \\
& \left.L_{x} \tilde{\phi}\right|_{x=0}=0, \quad y<0, t>0, \\
& \left.L_{y} \tilde{\phi}\right|_{y=0}=0, \quad x<0, t>0, \\
& \text { with proper initial conditions. }
\end{aligned}
$$

Well-posedness essentially requires that bounded solutions in space should not grow without a bound in time, as is stated in the second definition of well-posedness for the half-space problem. It is clear that therefore (7.1)-(7.4) can only be well-posed if each of the half-space problems is also well-posed:

$$
\begin{aligned}
& -\frac{\partial^{2} \tilde{\phi}}{\partial t^{2}}+c c_{g} \nabla^{2} \tilde{\phi}-\left(\omega^{2}-k^{2} c c_{g}\right) \tilde{\phi}=0, \\
& \quad x<0, y \in \mathbb{R}, t>0, \\
& \left.L_{x} \tilde{\phi}\right|_{x=0}=0, \quad y \in \mathbb{R}, t>0,
\end{aligned}
$$

with proper initial conditions,

and

$$
\begin{aligned}
& -\frac{\partial^{2} \tilde{\phi}}{\partial t^{2}}+c c_{g} \nabla^{2} \tilde{\phi}-\left(\omega^{2}-k^{2} c c_{g}\right) \tilde{\phi}=0, \\
& \quad x \in \mathbb{R}, y<0, t>0, \\
& \left.L_{y} \tilde{\phi}\right|_{y=0}=0, \quad x \in \mathbb{R}, t>0,
\end{aligned}
$$

with proper initial conditions.

This assures that the boundary conditions at the boundaries $\partial \Omega_{1}$ and $\partial \Omega_{2}$ do not admit any unstable solutions of the system. By continuity the field equation (7.1) and the boundary conditions (7.2) and (7.3) should be satisfied at the intersection point $O$. However, using these boundary conditions in $O$ as such, may cause instabilities, and some care has to be taken to impose the proper boundary condition(s) at the corner point.

For the first-order conditions $\mathrm{BC} 2$ at $\partial \Omega_{i}$, for instance, no special treatment is needed in $O$, but using the second-order condition BC3 will render the problem ill-posed.

This corner problem was also considered by Engquist and Majda in [12]. For the wave equation they give a boundary condition in $O$, which absorbs plane waves propagating along the diagonal $x=y$, and it is obvious that this idea can be used also for the Klein-Gordon equation.

Engquist and Majda give no arguments why this condition in $O$ renders the problem well-posed. Hence we still have to investigate when unstable solutions can be generated at the intersection point, because in general, the introduction of a boundary (the intersection point) of the boundary $\left(\partial \Omega_{i}\right)$ may cause unstable solutions to exist within the subspace of the boundary $\partial \Omega_{i}$, if this boundary $O$ of $\partial \Omega_{\imath}$ is not treated in a proper way.

Considering the subspaces $\partial \Omega_{i}$ we therefore demand that the IBVPs are also well-posed,

$$
\partial \Omega_{1}
$$

$\left.L_{y} \tilde{\phi}\right|_{y=0}=0, \quad x<0, t>0$,

with bound. cond. $\left.L_{y 0} \tilde{\phi}\right|_{x=y=0}=0, \quad t>0$,

and proper initial conditions,

and

$\partial \Omega_{2}$.

$\left.L_{x} \tilde{\phi}\right|_{x=0}=0, \quad y<0, t>0$,

with bound. cond. $\left.L_{x 0} \widetilde{\phi}\right|_{x=y=0}=0, \quad t>0$,

and proper initial conditions.

Thus the problems in the subspaces $\partial \Omega_{1}$ and $\partial \Omega_{2}$ should be investigated for well-posedness in the same way as the original IBVP in $\Omega$. We make the following

Proposition 1. (Sufficient condition for quarter-space problem). The quarter-space problem (7.1)-(7.4) is wellposed, if the half-space problems (7.5)-(7.7) and (7.8)-(7.10), as well as the subspace problems (7.11)-(7.13) and (7.14)-(7.16) are all well-posed.

The requirements given in the proposition are rather severe, and very likely they can be relaxed somewhat, but this is left for future study.

We will now use this proposition to study the well-posedness of some practical examples involving the absorbing boundary conditions BC1-BC4. 
EXAMPLE 1.

$$
\begin{array}{ll}
\partial \Omega_{1}: \frac{\partial \tilde{\phi}}{\partial t}+c \frac{\partial \tilde{\phi}}{\partial y}=0 & (\mathrm{BC} 2) \\
\partial \Omega_{2}: \frac{\partial \tilde{\phi}}{\partial t}+c \frac{\partial \tilde{\phi}}{\partial x}=0 & (\mathrm{BC} 2)
\end{array}
$$

These equations reduce to ordinary differential equations in the subspaces $\partial \Omega_{i}$, and no extra boundary conditions are needed in $O$.

EXAMPLE 2.

$$
\begin{aligned}
& \partial \Omega_{1}: a \cdot \frac{\partial^{2} \tilde{\phi}}{\partial t^{2}}+b \cdot \frac{\partial}{\partial t} \frac{\partial \tilde{\phi}}{\partial y} \\
& -c^{2} \cdot \frac{\partial^{2} \tilde{\phi}}{\partial x^{2}}+e \cdot \tilde{\phi}=0 \quad(\mathrm{BC} 4) \\
& \partial \Omega_{2}: \frac{\partial \tilde{\phi}}{\partial x}=0 \quad \text { (solid wall). }
\end{aligned}
$$

Assuming a periodic behaviour in normal $(y$-)direction, we can write $\bar{\phi}$ on $\partial \Omega_{1}$ as

$$
\bar{\phi}=e^{i \eta y} \cdot \psi(x, t)
$$

and substitute this into (7.19) to obtain the "field" equation for the subspace $\partial \Omega_{1}$,

$$
a \cdot \frac{\partial^{2} \psi}{\partial t^{2}}-c^{2} \cdot \frac{\partial^{2} \psi}{\partial x^{2}}+f \cdot \psi=0 \quad \text { on } \quad \partial \Omega_{1}
$$

which is a hyperbolic equation in $\psi$. This equation needs one boundary condition in $O$. This condition is provided by applying (7.20) in $O$, giving the boundary condition

$$
\frac{\partial \psi}{\partial x}=0 \quad \text { in } \quad O
$$

EXAMPLE 3.

$$
\begin{aligned}
& \partial \Omega_{1}: a \cdot \frac{\partial^{2} \tilde{\phi}}{\partial t^{2}}+b \cdot \frac{\partial}{\partial t} \frac{\partial \tilde{\phi}}{\partial y} \\
&-c^{2} \cdot \frac{\partial^{2} \tilde{\phi}}{\partial x^{2}}+e \cdot \tilde{\phi}=0 \quad(\mathrm{BC} 4), \\
& \partial \Omega_{2}: a \cdot \frac{\partial^{2} \tilde{\phi}}{\partial t^{2}}+b \cdot \frac{\partial}{\partial t} \frac{\partial \tilde{\phi}}{\partial x} \\
&-c^{2} \cdot \frac{\partial^{2} \tilde{\phi}}{\partial y^{2}}+e \cdot \tilde{\phi}=0 \quad \text { (BC4). }
\end{aligned}
$$

Now we need a boundary condition in $O$ for both equations.
In Example 2 the condition on the other boundary provided the extra boundary condition in $O$, but here this is not very convenient (especially in the discrete case) due to the second-order tangential derivatives.

Therefore it is more convenient to derive a new boundary condition to be used in $O$ to render the problem well-posed. Engquist and Majda's boundary condition for the wave equation is

$$
\sqrt{2} \frac{\partial \tilde{\phi}}{\partial t}+r\left(\frac{\partial \tilde{\phi}}{\partial x}+\frac{\partial \tilde{\phi}}{\partial y}\right)=0 \quad \text { in } \quad o .
$$

Substituting (7.21) in this equation results in the following boundary condition in $O$ for the hyperbolic problem on $\partial \Omega_{1}$ :

$$
\sqrt{2} \frac{\partial \psi}{\partial t}+c \frac{\partial \psi}{\partial x}+h \psi=0 \quad \text { in } \quad O
$$

This boundary condition renders the problem on $\partial \Omega_{1}$ wellposed. For reasons of symmetry (7.26) can be used for $\partial \Omega_{2}$ as well.

One can also use higher order conditions in $O$, e.g.,

$$
\begin{aligned}
& \left(\cos \left(\alpha_{1}\right) \frac{\partial}{\partial t}+c \frac{\partial}{\partial x}\right) \\
& \quad \times\left(\cos \left(\alpha_{2}\right) \frac{\partial}{\partial t}+c \frac{\partial}{\partial y}\right) \phi=0 \text { in } O .
\end{aligned}
$$

If the $\alpha_{i}$ are chosen in the interval $(0, \pi / 2)$, both subspace problems will be well-posed, giving a well-posed quarterspace problem.

\section{CONCLUSIONS}

In this paper, absorbing boundary conditions have been derived to be used on artificial boundaries in free surface flow problems. After a review of several techniques of absorbing free surface waves, approximations of exact pseudodifferential operators were given as first- and secondorder boundary conditions. The first-order condition BC1 results in weakly ill-posed IBVPs for all choices $\alpha$, whereas $\mathrm{BC} 2$, and the second-order conditions $\mathrm{BC} 3$ and $\mathrm{BC} 4$ lead to weakly ill-posed models only for $\alpha= \pm \pi / 2$ and to wellposedness in all other cases. Theoretically, well-posedness is not guaranteed in case corners are present, but the proposed conditions can be used in practical calculations, as is shown in the numerical experiments in $[39,4]$ and in the accompanying paper [5]. In the latter paper a discretization of the absorbing boundary conditions will be treated in detail. 


\section{ACKNOWLEDGMENTS}

The investigations were supported by the Netherlands Technology Foundation (STW), by Delft Hydraulics, and by the University of Twente.

\section{REFERENCES}

1. G. R. Baker, D. I. Meiron, and S. A. Orszag, in Proceedings, Third International Conference on Numerical Ship Hydrodynamics, Paris, 1981, edited by J. C. Dern et al. (Bassin d'Essais des Carènes, Paris, $1981)$, p. 179.

2. A. Bayliss, M. Gunzburger, and E. Turkel, SIAM J. Appl. Math. 42 , 430 (1982).

3. P. L. Betts and T. T. Mohamad, in Proceedings, Fourth International Symposium on Finite Element Methods in Flow Problems, Tokyo, 1982, edited by T. Kawai (Univ. of Tokyo Press, Tokyo; North-Holland, Amsterdam, 1982), p. 923.

4. J. Broeze, Delft Hydraulics, Report H475, Marknesse, 1988 (unpublished).

5. J. Broeze and J. E. Romate, Absorbing boundary conditions for free surface wave simulations with a panel method, J. Comput. Phys., submitted.

6. R. K.-C. Chan, in Proceedings, first International Conference on Numerical Ship Hydrodynamics, Bethesda, Maryland, 1975, edited by J. W. Schot et al., p. 315.

7. R. K.-C. Chan, in Proceedings, second International Conference on Numerical Ship Hydrodynamics, Berkeley, California., 1977, edited by J. V. Wehausen et al. (Univ. Extension Publ., Berkeley, 1977), p. 39.

8. R. Courant and D. Hilbert, Methods of Mathematical Physics, II (Interscience, New York, 1962).

9. M. W. Dingemans, Delft Hydraulics, Rep. W301, part 5, Delft, 1985 (unpublished).

10. D. G. Dommermuth and D. K. P. Yuc, J. Fluid Mech 178, 195 (1987).

11. B. Engquist and A. Majda, Math. Comput. 31, 629 (1977).

12. B. Engquist and A. Majda, Commun. Pure Appl. Math. 32, 313 (1979).

13. T. D. Finnigan and T. Yamamoto, in Proceedings, 15 th Coastal Eng. Conf., Vol. III, Honolulu, 1976 (Am. Soc. of Civil Eng., New York, 1976), p. 380.

14. H. Gerritsen, Ph.D. thesis, University of Twente, Enschede, 1982.

15. R. L. Higdon, Math. Comput. 47, 437 (1986).
16. R. L. Higdon, SIAM Rev. 28, 177 (1986).

17. M. de St. Q. Isaacson, J. Fluid Mech. 120, 267 (1982).

18. M. Israeli and S. A. Orszag, J. Comput. Phys. 41, 115 (1981).

19. H.-O. Kreiss, Commun. Pure Appl. Math. 23, 277 (1970).

20. J. Larsen and H. Dancy, Coastal Eng. 7, 285 (1983).

21. G. P. Lennon, Ph. L.-F. Liu, and J. A. Liggett, J. Hydraulics Div., Proc. ASCE 108, 921 (1982).

22. W.-M. Lin, J. N. Newman, and D. K. Yue, in Proceedings, 15th Symp. on Naval Hydrodynamics, IIamburg, 1984 (National Academy Press, Washington, 1985), p. 1.

23. Ph. L.-F. Liu and M. Abbaspour, Int. I. Numer. Methods Fng. 18, 1497 (1982).

24. M. S. Longuet-Higgins and E. D. Cokelet, Proc. R. Soc. London A 350 , 1 (1976).

25. C. C. Mei, The Applied Dynamics of Ocean Surface Waves (Wiley, New York, 1983).

26. A. Nestegard and P. D. Sclavounos, J. Ship Res. 28, 48 (1984).

27. I. Orlanski, J. Comput. Phys. 21, 251 (1976).

28. J. E. Romate, Delft Hydraulics, Rep. S174, Delft, 1984 (unpublished).

29. J. E. Romate, Deltt Hydraulics, Memorandum H463.06, Markness, 1985 (unpublished).

30. J. E. Romate, Ph. D. thesis, University of Twente, Enschede, 1989.

31. J. E. Romate and P. J. Zandbergen, in Advanced Boundary Element Methods, edited by Th. A. Cruse (Springer-Verlag, Berlin, 1988), p. 359 .

32. A. Sommerfeld, Partial Differential Equations in Physics (Academic Press, New York, 1949).

33. T.-K. Tsay and Ph. L.-F. Liu, Appl. Ocean Res. 5, 30 (1983).

34. G. K. Verboom, Delft Hydraulics, Publication 266, Delft, 1982.

35. R. Vichnevetsky and E. C. Parisier, Comput. Math. Appl. 11, 67 (1985).

36. T. Vinje and P. Brevig, in Proceedings, Int. Symp. on Hydrodynamics in Ocean Engineering, Tronaheim, 1981, (Norwegian Hydrodynamics Laboratories, Trondheim, 1981), p. 547.

37. L. Wagatha, Numer. Math. 42, 51 (1983).

38. G. B. Whitham, Linear and Nonlinear Waves (Wiley-Interscience, New York, 1974).

39. S. M. Yen and D. R. Hall, in Proceedings, third International Conference on Numerical Ship Hydrodynamics, Paris, 1981, edited by J. C. Dern et al. (Bassin d'Essais des Carènes, Paris, 1981), p. 163.

40. R. W. Yeung, Ann. Rev. Fluid Mech. 14, 395 (1982). 